\title{
EXTREME POINTS IN SPACES OF POLYNOMIALS
}

\author{
Konstantin M. Dyakonov
}

\begin{abstract}
We determine the extreme points of the unit ball in spaces of complex polynomials (of a fixed degree), living either on the unit circle or on a subset of the real line and endowed with the supremum norm.
\end{abstract}

\section{Introduction}

Let $\mathcal{P}_{n}$ stand for the space of all polynomials with complex coefficients of degree not exceeding $n$. Given a compact set $E \subset \mathbb{C}$, one may treat $\mathcal{P}_{n}$ as a subspace of $C(E)$, the space of continuous functions on $E$, and equip it with the maximum norm

$$
\|P\|_{\infty}=\|P\|_{\infty, E}:=\max _{z \in E}|P(z)| \quad\left(P \in \mathcal{P}_{n}\right) .
$$

The resulting space will be denoted by $\mathcal{P}_{n}(E)$. We write

$$
\operatorname{ball}\left(\mathcal{P}_{n}(E)\right):=\left\{P \in \mathcal{P}_{n}:\|P\|_{\infty, E} \leq 1\right\}
$$

for the unit ball of $\mathcal{P}_{n}(E)$, and we shall be concerned with the extreme points of this ball. (As usual, an element of a convex set $S$ is said to be its extreme point if it is not the midpoint of any nondegenerate segment contained in $S$.)

In this paper, we explicitly characterize the extreme points of ball $\left(\mathcal{P}_{n}(E)\right)$ in the case where $E$ is either the circle $\mathbb{T}:=\{z \in \mathbb{C}:|z|=1\}$ or a perfect compact subset of the real line $\mathbb{R}$. The description obtained is, perhaps, a bit more complicated than one could at first expect; however, the complexity seems to be in the nature of things.

Let us begin by recalling that the extreme points of the unit ball in $L^{\infty}(\mathbb{T})-$ or in $C(\mathbb{T})$ - are precisely the functions of modulus 1 . (The same applies to other sets in place of $\mathbb{T}$.) Further, in the space $H^{\infty}$ of bounded analytic functions on $\{|z|<1\}$, as well as in the disk algebra $H^{\infty} \cap C(\mathbb{T})$, the extreme points are known to be the unit-norm functions $f$ with $\int_{\mathbb{T}} \log \left(1-|f(z)|^{2}\right)|d z|=-\infty$; see [H, Chap. 9].

Received September 30, 2002.

2000 Mathematics Subject Classification. 46B20, 46E30

Supported in part by Grant 02-01-00267 from the Russian Foundation for Fundamental Research, by a PIV fellowship from Generalitat de Catalunya, and by the Ramón y Cajal program (Spain). 
Yet another relevant example is provided by a theorem of Konheim and Rivlin $[\mathrm{KR}]$, dealing with the space $\mathcal{P}_{n}^{\mathbb{R}}(I)$ of all real polynomials of degree $\leq n$ on the segment $I:=[-1,1]$. The theorem states that a unit-norm polynomial $P$ is an extreme point of ball $\left(\mathcal{P}_{n}^{\mathbb{R}}(I)\right)$ if and only if $\mathcal{N}_{I}\left(1-P^{2}\right)>n$; here $\mathcal{N}_{I}(f)$ is the total number of zeros (multiplicities included) that $f$ has on $I$. A similar result holds for real trigonometric polynomials on $\mathbb{T}$; see $[R]$ or Proposition 1 in Section 1 below.

With these examples in mind, one might be tempted to believe that, in order to recognize the extreme points among all unit-norm elements $P$ of the complex space $\mathcal{P}_{n}(E)$ (say, with $E=\mathbb{T}$ or $E=I$ ), one only needs to know "how often" $|P|$ takes the extremal value 1 on $E$. In other words, one might seek to characterize the extreme points $P$ in terms of the zeros - and their multiplicities - of the polynomial $1-|P|^{2}$. (Strictly speaking, $1-|P|^{2}$ is a trigonometric polynomial for $P \in \mathcal{P}_{n}(\mathbb{T})$ and a true polynomial when $P$ lives on $\mathbb{R}$.)

However, no such thing can be done. Indeed, along with solving the two versions of the problem in Sections 1 and 2 (one of these deals with the circle, and the other with subsets of $\mathbb{R}$ ), we also construct in each case a pair of unitnorm polynomials $P_{1}, P_{2}$ in $\mathcal{P}_{n}(E)$ satisfying

$$
1-\left|P_{1}\right|^{2}=2\left(1-\left|P_{2}\right|^{2}\right)
$$

so that $P_{1}$ is a non-extreme point of $\operatorname{ball}\left(\mathcal{P}_{n}(E)\right)$, while $P_{2}$ is extreme. In fact, the construction is carried out for the smallest possible value of $n$, which equals 2 when $E=\mathbb{T}$, and 3 when $E$ is a real segment.

In conclusion, we briefly mention the $L^{1}$ counterpart of the problem, i.e., the problem of determining the extreme points of the unit ball in certain $L^{1}$-spaces of polynomials. Here, the real case was settled by Garkavi $[\mathrm{G}]$ and the complex case by the author [D]. Garkavi's, as well as Konheim and Rivlin's results were then rediscovered - or reproved - by Parnes in $[\mathrm{P}]$, where the current problem (the case of complex polynomials on $\mathbb{T}$ with the sup-norm) was also considered, but not solved.

I thank Evgeny Abakumov for bringing Parnes' work to my attention.

\section{Polynomials on the circle}

Among the unit-norm polynomials in $\mathcal{P}_{n}(\mathbb{T})$, we single out the class of monomials; these are of the form $c z^{k}$, where $c \in \mathbb{C},|c|=1$ and $0 \leq k \leq n$. Of course, every monomial is an extreme point of $\operatorname{ball}\left(\mathcal{P}_{n}(\mathbb{T})\right)$.

Now if $P \in \mathcal{P}_{n}(\mathbb{T})$ satisfies $\|P\|_{\infty}=1$ and is distinct from a monomial, let $z_{1}, \ldots, z_{N}$ be an enumeration of the (nonempty) set $\{z \in \mathbb{T}:|P(z)|=1\}$. The points $z_{1}, \ldots, z_{N}$ are thus the distinct zeros of $1-|P|^{2}$ lying on $\mathbb{T}$, and the multiplicities of these zeros will be denoted by $2 \mu_{1}, \ldots, 2 \mu_{N}$. The $\mu_{j}$ 's are positive integers, and their sum

$$
\mu:=\sum_{j=1}^{N} \mu_{j}
$$


does not exceed $n$. To see why, note that the function $z \mapsto 1-|P(z)|^{2}$ (living on $\mathbb{T}$ ) is a nonnegative trigonometric polynomial of degree $\leq n$, not vanishing identically. Therefore, its zeros lying on $\mathbb{T}$ are necessarily of even order, while the total number of its zeros (multiplicities included) is at most $2 n$. Hence $2 \mu_{1}+\cdots+2 \mu_{N} \leq 2 n$, so that $\mu \leq n$, as claimed above.

Next, for $z=e^{i t} \in \mathbb{T}$ and $k \in \mathbb{N}$, consider the Wronski-type matrix

$$
W(z ; k)=\left(\begin{array}{cccc}
\bar{z}^{\mu / 2} P(z) & \bar{z}^{\mu / 2+1} P(z) & \cdots & \bar{z}^{n-\mu / 2} P(z) \\
\left(\bar{z}^{\mu / 2} P(z)\right)^{\prime} & \left(\bar{z}^{\mu / 2+1} P(z)\right)^{\prime} & \cdots & \left(\bar{z}^{n-\mu / 2} P(z)\right)^{\prime} \\
\cdots & \ldots & \ldots & \ldots \\
\left(\bar{z}^{\mu / 2} P(z)\right)^{(k-1)} & \left(\bar{z}^{\mu / 2+1} P(z)\right)^{(k-1)} & \cdots & \left(\bar{z}^{n-\mu / 2} P(z)\right)^{(k-1)}
\end{array}\right)
$$

The exponent $n-\mu / 2$ in the last column should be viewed as $\mu / 2+(n-\mu)$; thus, $W(z ; k)$ is a $k \times(n-\mu+1)$ matrix. The derivatives involved are with respect to the real variable $t=\arg z$.

Let $W_{\mathfrak{R}}(z ; k)$ and $W_{\mathfrak{I}}(z ; k)$ stand for the real and imaginary parts of $W(z ; k)$, respectively. Finally, we need the block matrix

$$
W_{P}=\left(\begin{array}{cc}
W_{\mathfrak{R}}\left(z_{1} ; \mu_{1}\right) & W_{\mathfrak{I}}\left(z_{1} ; \mu_{1}\right) \\
W_{\mathfrak{R}}\left(z_{2} ; \mu_{2}\right) & W_{\mathfrak{I}}\left(z_{2} ; \mu_{2}\right) \\
\cdots & \cdots \\
W_{\mathfrak{R}}\left(z_{N} ; \mu_{N}\right) & W_{\mathfrak{I}}\left(z_{N} ; \mu_{N}\right)
\end{array}\right)
$$

Here, each "entry" $W_{\mathfrak{R}}\left(z_{j} ; \mu_{j}\right)$ or $W_{\mathfrak{I}}\left(z_{j} ; \mu_{j}\right)$ is actually a $\mu_{j} \times(n-\mu+1)$ submatrix, as defined above, where everything is computed at the point $z_{j}$. In particular, $W_{P}$ is a $\mu \times 2(n-\mu+1)$ matrix, and its rank is therefore bounded by $\min (\mu, 2(n-\mu+1))$.

Theorem 1. Let $P \in \mathcal{P}_{n}(\mathbb{T}),\|P\|_{\infty}=1$. The following are equivalent.

(i) $P$ is an extreme point of $\operatorname{ball}\left(\mathcal{P}_{n}(\mathbb{T})\right)$.

(ii) Either $P$ is a monomial, or $\operatorname{rank} W_{P}=2(n-\mu+1)$.

The proof will be preceded by a brief discussion.

First of all, the condition $\operatorname{rank} W_{P}=2(n-\mu+1)$ can only be met if $\mu \geq$ $2(n-\mu+1)$, i.e., if $\mu \geq \frac{2}{3}(n+1)$. (The weaker condition $\mu>n / 2$ was pointed out in $[\mathrm{P}]$ as necessary in order that $P$ be an extreme point.) The inequalities $\frac{2}{3}(n+1) \leq \mu \leq n$ being incompatible for $n=0$ and $n=1$, there are no nontrivial extreme points for these $n$. (Here and below, "nontrivial" means "distinct from a monomial".) Now for $n=2,3,4$, the two inequalities - in conjunction with the fact that $\mu \in \mathbb{N}$ - reduce to the condition $\mu=n$, which must be therefore fulfilled by each nontrivial extreme point $P$ of $\operatorname{ball}\left(\mathcal{P}_{n}(\mathbb{T})\right)$.

On the other hand, for $n \geq 2$, the nontrivial extreme points $P$ with $\mu=n$ are characterized by the condition $\operatorname{rank} W_{P}=2$, which means that the two columns of $W_{P}$ are linearly independent. This, in turn, is equivalent to saying that there 
is no straight line in $\mathbb{C}$ passing through the origin and containing the set

$$
\bigcup_{j=1}^{N}\left\{\bar{z}_{j}^{n / 2} P\left(z_{j}\right),\left(\bar{z}^{n / 2} P\right)^{\prime}\left(z_{j}\right), \ldots,\left(\bar{z}^{n / 2} P\right)^{\left(\mu_{j}-1\right)}\left(z_{j}\right)\right\} .
$$

Now let us consider an example.

Example 1. Put $P_{0}(z):=\frac{1}{2}\left(z+z^{-1}\right)$, so that $P_{0}\left(e^{i t}\right)=\cos t$; then define

$$
P_{1}(z):=z P_{0}(z)=\frac{1}{2}\left(z^{2}+1\right)
$$

and

$$
P_{2}(z):=\frac{z}{\sqrt{2}}\left(P_{0}(z)+i\right)=\frac{1}{2 \sqrt{2}}\left(z^{2}+2 i z+1\right) .
$$

Clearly, $P_{1}$ and $P_{2}$ are unit-norm polynomials in $\mathcal{P}_{2}(\mathbb{T})$. In fact, for $z=e^{i t} \in \mathbb{T}$,

$$
\left|P_{1}(z)\right|^{2}=P_{0}^{2}(z)=\cos ^{2} t
$$

and

$$
\left|P_{2}(z)\right|^{2}=\frac{1}{2}\left(P_{0}^{2}(z)+1\right)=\frac{1}{2}\left(\cos ^{2} t+1\right) .
$$

In particular,

$$
1-\left|P_{1}(z)\right|^{2}=2\left(1-\left|P_{2}(z)\right|^{2}\right), \quad z \in \mathbb{T}
$$

(indeed, both sides equal $\sin ^{2} t$ ), and so the two polynomials have the same $z_{j}$ 's and $\mu_{j}$ 's. Specifically, these are $z_{1}=1, z_{2}=-1$ (or vice versa) and $\mu_{1}=\mu_{2}=1$, so that $N=\mu=n=2$.

However, while $P_{1}$ is the arithmetic mean of two monomials, $z^{2}$ and 1 , and hence a non-extreme point of $\operatorname{ball}\left(\mathcal{P}_{2}(\mathbb{T})\right)$, it turns out that $P_{2}$ is an extreme point thereof. This last fact follows by Theorem 1 , since the matrix

$$
W_{P_{2}}=\left(\begin{array}{cc}
\mathfrak{R}\left(P_{2}(1)\right) & \mathfrak{I}\left(P_{2}(1)\right) \\
\mathfrak{R}\left(-P_{2}(-1)\right) & \mathfrak{I}\left(-P_{2}(-1)\right)
\end{array}\right)=\frac{1}{\sqrt{2}}\left(\begin{array}{cc}
1 & 1 \\
-1 & 1
\end{array}\right)
$$

has rank 2 .

The proof of Theorem 1 will rely on two elementary observations. The first of these, stated for an arbitrary compact set $E \subset \mathbb{C}$, will also be used when proving Theorem 2 in the next section.

Observation 1. Clearly, a given unit-norm polynomial $P \in \mathcal{P}_{n}$ is an extreme point of ball $\left(\mathcal{P}_{n}(E)\right)$ if and only if the only polynomial $Q \in \mathcal{P}_{n}$ satisfying

$$
\|P+Q\|_{\infty} \leq 1 \text { and }\|P-Q\|_{\infty} \leq 1
$$


is $Q \equiv 0$. Rewriting (1.1) as

$$
|P \pm Q|^{2}=|P|^{2} \pm 2 \Re(\bar{P} Q)+|Q|^{2} \leq 1
$$

and noting that $\max (x,-x)=|x|$ for all $x \in \mathbb{R}$, we see that $P$ is extreme iff there is no nontrivial $Q \in \mathcal{P}_{n}$ for which

$$
2|\Re(\bar{P} Q)|+|Q|^{2} \leq 1-|P|^{2}
$$

everywhere on $E$.

Observation 2. If $z=e^{i t}$ and $\zeta_{j}=e^{i t_{j}}(j=1, \ldots, N)$ are points of $\mathbb{T}$, and if $k_{1}, \ldots, k_{N}$ are positive integers with $\sum_{j=1}^{N} k_{j}=k$, then the identities

$$
z-\zeta_{j}=2 i e^{i t_{j} / 2} e^{i t / 2} \sin \frac{t-t_{j}}{2}
$$

yield

$$
\prod_{j=1}^{N}\left(z-\zeta_{j}\right)^{k_{j}}=c e^{i k t / 2} \prod_{j=1}^{N}\left(\sin \frac{t-t_{j}}{2}\right)^{k_{j}}
$$

where

$$
c=(2 i)^{k} \prod_{j=1}^{N} \exp \left(\frac{i k_{j} t_{j}}{2}\right) .
$$

Proof of Theorem 1. (ii) $\Longrightarrow$ (i). We shall assume that $P$ is distinct from a monomial (otherwise, it is obviously extreme) and that rank $W_{P}=2(n-\mu+1)$. Now suppose (1.2) holds for some $Q \in \mathcal{P}_{n}$. In particular, we have then

$$
|Q(z)|^{2} \leq 1-|P(z)|^{2}, \quad z \in \mathbb{T}
$$

and so, for $j=1, \ldots, N$, the polynomial $Q$ vanishes at $z_{j}$ with multiplicity at least $\mu_{j}$. (Recall that the multiplicity of $z_{j}$ as a zero of $1-|P|^{2}$ is $2 \mu_{j}$.) Hence $Q$ is divisible by $\prod_{j=1}^{N}\left(z-z_{j}\right)^{\mu_{j}}$ and takes the form

$$
Q(z)=Q_{0}(z) \cdot z^{\mu / 2} \prod_{j=1}^{N}\left(\sin \frac{t-t_{j}}{2}\right)^{\mu_{j}}, \quad z=e^{i t} \in \mathbb{T},
$$

for some $Q_{0} \in \mathcal{P}_{n-\mu}$; by $t_{j}$ we now denote $\arg z_{j}$. Here, to arrive at (1.4), we have used (1.3) with $z_{j}$ in place of $\zeta_{j}$ and with $\mu_{j}$ (resp., $\mu$ ) in place of $k_{j}$ (resp., $k)$. From (1.4) we get

$$
\mathfrak{R}(\bar{P}(z) Q(z))=\prod_{j=1}^{N}\left(\sin \frac{t-t_{j}}{2}\right)^{\mu_{j}} \mathfrak{R}\left(z^{\mu / 2} \bar{P}(z) Q_{0}(z)\right) .
$$


Combining this with the fact that

$$
|\mathfrak{R}(\bar{P}(z) Q(z))| \leq 1-|P(z)|^{2}
$$

(which is contained in (1.2)) yields

$$
\left|\mathfrak{R}\left(z^{\mu / 2} \bar{P}(z) Q_{0}(z)\right)\right| \leq\left(1-|P(z)|^{2}\right) \prod_{j=1}^{N}\left|\sin \frac{t-t_{j}}{2}\right|^{-\mu_{j}}, \quad z=e^{i t} \in \mathbb{T} .
$$

The right-hand side of (1.6) being $O\left(\left|z-z_{j}\right|^{\mu_{j}}\right)$ as $z \rightarrow z_{j}$, the left-hand side must also vanish at each $z_{j}$ with multiplicity at least $\mu_{j}$. In other words, for each $j=1, \ldots, N$, one has

$$
\mathfrak{R}\left(z^{\mu / 2} \bar{P} Q_{0}\right)^{(l)}\left(z_{j}\right)=0 \quad\left(l=0,1, \ldots, \mu_{j}-1\right) .
$$

Putting

$$
Q_{0}(z)=\sum_{k=0}^{n-\mu}\left(c_{k}+i d_{k}\right) z^{k}
$$

and substituting this into (1.7), we obtain

$$
\begin{gathered}
\sum_{k=0}^{n-\mu} c_{k} \mathfrak{R}\left(z^{\mu / 2+k} \bar{P}\right)^{(l)}\left(z_{j}\right)-\sum_{k=0}^{n-\mu} d_{k} \mathfrak{I}\left(z^{\mu / 2+k} \bar{P}\right)^{(l)}\left(z_{j}\right)=0 \\
\left(j=1, \ldots, N ; l=0,1, \ldots, \mu_{j}-1\right),
\end{gathered}
$$

which can be viewed as a homogeneous system of $\mu_{1}+\cdots+\mu_{N}=\mu$ linear equations with $2(n-\mu+1)$ real unknowns $c_{0}, \ldots, c_{n-\mu}, d_{0}, \ldots, d_{n-\mu}$. The matrix of this system is precisely $W_{P}$, and the hypothesis rank $W_{P}=2(n-\mu+1)$ implies that the only solution is

$$
c_{0}=\cdots=c_{n-\mu}=d_{0}=\cdots=d_{n-\mu}=0 .
$$

Thus $Q_{0} \equiv 0$, whence also $Q \equiv 0$, and $P$ is an extreme point.

(i) $\Longrightarrow$ (ii). The above argument can be essentially reversed. Indeed, suppose that (ii) fails, so that $P$ is distinct from a monomial and $\operatorname{rank} W_{P}<2(n-\mu+1)$. The homogeneous system (1.8) has then a nontrivial solution, and the equations (1.7) hold for $j=1, \ldots, N$ with some $Q_{0} \in \mathcal{P}_{n-\mu}, Q_{0} \not \equiv 0$. Multiplying $Q_{0}$ by a number $\varepsilon>0$ (if necessary), we may assume in addition that the norm $\left\|Q_{0}\right\|_{\infty}$ is appropriately small; we shall specify our choice later.

Now that we have such a $Q_{0}$ at our disposal, let us define $Q$ by (1.4), where, as before, it is understood that $z_{j}=e^{i t_{j}}$. By Observation 2, we have $Q \in \mathcal{P}_{n}$; we also remark that $Q \not \equiv 0$, because $Q_{0} \not \equiv 0$, and that (1.5) holds true. 
We further claim that

$$
|Q(z)|^{2}=O\left(1-|P(z)|^{2}\right), \quad z \in \mathbb{T}
$$

and

$$
|\mathfrak{R}(\bar{P}(z) Q(z))|=O\left(1-|P(z)|^{2}\right), \quad z \in \mathbb{T}
$$

Indeed, (1.9) is fulfilled because $Q$ is divisible by $\prod_{j=1}^{N}\left(z-z_{j}\right)^{\mu_{j}}$, and so $|Q(z)|^{2}$ vanishes at those points of $\mathbb{T}$ (viz., $z_{1}, \ldots, z_{N}$ ) where $1-|P(z)|^{2}$ does, with at least the same multiplicities (viz., $2 \mu_{1}, \ldots, 2 \mu_{N}$ ). Similarly, to verify (1.10), one checks that its left-hand side has a zero at each $z_{j}$ of multiplicity $\geq 2 \mu_{j}$; this is due to (1.5) and (1.7).

In view of the above discussion, we could have started with a $Q_{0}$ for which the quantity $\left\|Q_{0}\right\|_{\infty}$, and hence also the "big oh" constants in (1.9) and (1.10), are as small as desired. In particular, a suitable choice ensures that

$$
|Q(z)|^{2} \leq \frac{1}{2}\left(1-|P(z)|^{2}\right), \quad z \in \mathbb{T}
$$

and

$$
2|\Re(\bar{P}(z) Q(z))| \leq \frac{1}{2}\left(1-|P(z)|^{2}\right), \quad z \in \mathbb{T} .
$$

Summing, we arrive at (1.2) and conclude that $P$ is not an extreme point. The proof is complete.

One might also consider the space $\mathcal{T}_{n}$ of all trigonometric polynomials of degree $\leq n$; these are, by definition, functions of the form $\sum_{k=-n}^{n} c_{k} z^{k}$ living on $\mathbb{T}$. A trigonometric polynomial $T \in \mathcal{T}_{n}$ is an extreme point of ball $\left(\mathcal{T}_{n}\right)$ if and only if $z^{n} T$ is an extreme point of ball $\left(\mathcal{P}_{2 n}\right)$. Thus, the extreme points $T$ of ball $\left(\mathcal{T}_{n}\right)$ are actually described by Theorem 1 , where obvious adjustments are needed: one should first replace $n$ by $2 n$, and then $P$ by $z^{n} T$. (Of course, the monomials in the theorem's statement should now include those with negative exponents, too.)

Finally, we briefly discuss the subspace $\mathcal{T}_{n}^{\mathbb{R}}$ of real-valued functions in $\mathcal{T}_{n}$; a trigonometric polynomial $\sum_{k=-n}^{n} c_{k} z^{k}$ is thus in $\mathcal{T}_{n}^{\mathbb{R}}$ iff $c_{-k}=\bar{c}_{k}$ for $|k| \leq n$. As before, given a nonconstant $P \in \mathcal{T}_{n}^{\mathbb{R}}$ with $\|P\|_{\infty}=1$, we let $z_{j}=e^{i t_{j}}(j=$ $1, \ldots, N$ ) be the distinct zeros that the (nonnegative) trigonometric polynomial $1-P^{2}$ happens to have on $\mathbb{T}$; the (even) multiplicity of the zero $z_{j}$ is again denoted by $2 \mu_{j}$, and we write $\mu=\sum_{j=1}^{N} \mu_{j}$. This time, however, $1-P^{2}$ is of degree $\leq 2 n$, so the only a priori estimate on $\mu$ is that $\mu \leq 2 n$. As to the constant polynomials $P \equiv 1$ and $P \equiv-1$, for each of these we put $\mu=+\infty$.

The following proposition is a trigonometric version of the Konheim-Rivlin result that can be found in $[R]$; a short self-contained proof will be given here for the sake of completeness. 
Proposition 1. Let $P \in \mathcal{T}_{n}^{\mathbb{R}},\|P\|_{\infty}=1$. Then $P$ is an extreme point of ball $\left(\mathcal{T}_{n}^{\mathbb{R}}\right)$ if and only if $\mu>n$.

Proof. To prove the "if" part, assume that $\mu>n$ and that (1.1) holds for some $Q \in \mathcal{T}_{n}^{\mathbb{R}}$. We have then $\pm P \pm Q \leq 1$ on $\mathbb{T}$, where the signs can be chosen in the four possible ways. Consequently,

$$
|Q| \leq 1-|P| \leq 1-P^{2} .
$$

Now since the right-hand side has in total $2 \mu(>2 n)$ zeros on $\mathbb{T}$, while $Q$ is of degree $\leq n$, it follows that $Q \equiv 0$ and $P$ is an extreme point.

To establish the "only if" part, assume that $\mu \leq n$ and put

$$
Q\left(e^{i t}\right):=\varepsilon \prod_{j=1}^{N}\left(\sin \frac{t-t_{j}}{2}\right)^{2 \mu_{j}},
$$

with a suitable $\varepsilon>0$. Then $Q \in \mathcal{T}_{n}^{\mathbb{R}}$, and making $\varepsilon$ sufficiently small we can arrange it so that

$$
|Q| \leq \frac{1}{2}\left(1-P^{2}\right) \leq 1-|P|
$$

From this, (1.1) follows immediately, and $P$ fails to be extreme.

We remark, in conclusion, that every nonconstant trigonometric polynomial in ball $\left(\mathcal{T}_{n}^{\mathbb{R}}\right)$ is a non-extreme point of ball $\left(\mathcal{T}_{n}\right)$, the unit ball of the complex space $\mathcal{T}_{n}$.

\section{Polynomials on subsets of $\mathbb{R}$}

Let $K$ be a perfect compact subset of $\mathbb{R}$ (as usual, "perfect" means "having no isolated points"), and let $P$ be a nonconstant polynomial in $\mathcal{P}_{n}(K)$ with $\|P\|_{\infty}=1$. Here and throughout this section, $\|P\|_{\infty}$ stands for $\|P\|_{\infty, K}:=$ $\max _{x \in K}|P(x)|$. (Likewise, some of the other symbols below should not be confused with their namesakes in Section 1.)

Further, let $x_{1}, \ldots, x_{N}$ be the distinct elements of the set $\{x \in K:|P(x)|=$ $1\}$, and let $m_{1}, \ldots, m_{N}$ denote the respective multiplicities of these points, regarded as zeros for $1-|P|^{2}$. (We remark that $m_{j}$ need not be even, unless $x_{j}$ is an interior point for $K$.) The function $x \mapsto 1-|P(x)|^{2}$ being a polynomial of degree $\leq 2 n$, we have $m_{1}+\cdots+m_{N} \leq 2 n$. Next, we introduce the numbers

$$
\mu_{j}:=\left[\frac{m_{j}+1}{2}\right] \quad(j=1, \ldots, N),
$$

where [·] denotes the integral part, and their sum $\mu:=\sum_{j=1}^{N} \mu_{j}$. Finally, let $M$ be the number of those $j$ 's for which $m_{j} \geq 2$. Thus $0 \leq M \leq N$, and we may assume that the inequality $m_{j} \geq 2$ holds precisely for $1 \leq j \leq M$. 
For a constant polynomial $P \equiv c$ with $|c|=1$, we put $\mu=+\infty$.

Now suppose $P$ is a unit-norm polynomial in $\mathcal{P}_{n}(K)$ with the property $\mu \leq n$. To such a $P$, we associate the Wronski-type matrix

$$
W(x ; k)=\left(\begin{array}{cccc}
P(x) & x P(x) & \cdots & x^{n-\mu} P(x) \\
P^{\prime}(x) & (x P(x))^{\prime} & \cdots & \left(x^{n-\mu} P(x)\right)^{\prime} \\
\cdots & \cdots & \cdots & \cdots \\
P^{(k-1)}(x) & (x P(x))^{(k-1)} & \cdots & \left(x^{n-\mu} P(x)\right)^{(k-1)}
\end{array}\right),
$$

where $x \in \mathbb{R}$ and $k \in \mathbb{N}$. The real and imaginary parts of $W(x ; k)$ will be denoted by $W_{\mathfrak{R}}(x ; k)$ and $W_{\mathfrak{I}}(x ; k)$. This said, we form the block matrix

$$
W_{P}=\left(\begin{array}{cc}
W_{\mathfrak{R}}\left(x_{1} ; m_{1}-\mu_{1}\right) & W_{\mathfrak{I}}\left(x_{1} ; m_{1}-\mu_{1}\right) \\
W_{\mathfrak{R}}\left(x_{2} ; m_{2}-\mu_{2}\right) & W_{\mathfrak{I}}\left(x_{2} ; m_{2}-\mu_{2}\right) \\
\cdots & \cdots \\
W_{\mathfrak{R}}\left(x_{M} ; m_{M}-\mu_{M}\right) & W_{\mathfrak{I}}\left(x_{M} ; m_{M}-\mu_{M}\right)
\end{array}\right)
$$

which has $\sum_{j=1}^{N} m_{j}-\mu$ rows and $2(n-\mu+1)$ columns. In the case that $M=0$ (i.e., when $m_{j}=\mu_{j}=1$ for all $j$ ), it is understood that $W_{P}$ is the zero matrix (of any order), so that $\operatorname{rank} W_{P}=0$.

Theorem 2. Let $P \in \mathcal{P}_{n}(K)$ and $\|P\|_{\infty}=1$. The following are equivalent.

(i) $P$ is an extreme point of ball $\left(\mathcal{P}_{n}(K)\right)$.

(ii) Either $\mu>n$, or $\operatorname{rank} W_{P}=2(n-\mu+1)$.

One easily checks that for $n \leq 2$, condition (ii) reduces to just saying that $\mu>n$. It is for $n \geq 3$ that things become more complicated, as the following example shows.

Example 2. Let $K=[-1,2]$, and put

$$
P_{1}(x):=\frac{1}{2}\left(x^{3}-3 x\right), \quad P_{2}(x):=\frac{1}{\sqrt{2}}\left(P_{1}(x)+i\right) .
$$

One easily verifies that $\left|P_{1}(x)\right| \leq 1$ for $x \in K$, the equality being attained at the points

$$
x_{1}=-1, \quad x_{2}=1, \quad x_{3}=2 .
$$

Then one deduces a similar fact for $P_{2}$ by noting that

$$
\left|P_{2}(x)\right|^{2}=\frac{1}{2}\left(P_{1}^{2}(x)+1\right) .
$$

Thus, $P_{1}$ and $P_{2}$ are unit-norm elements of $\mathcal{P}_{3}(K)$. Furthermore,

$$
1-P_{1}^{2}(x)=2\left(1-\left|P_{2}(x)\right|^{2}\right)=-\frac{1}{4}(x+2)(x+1)^{2}(x-1)^{2}(x-2) .
$$


The zeros of this last polynomial belonging to $K$ (i.e., the common $x_{j}$ 's for $P_{1}$ and $\left.P_{2}\right)$ are given by (2.1), and the corresponding (common) multiplicities are

$$
m_{1}=2, \quad m_{2}=2, \quad m_{3}=1 .
$$

Hence $\mu_{1}=\mu_{2}=\mu_{3}=1$, so that $N=\mu=n=3$ and $M=2$. Theorem 2 tells us now that $P_{1}$ is a non-extreme point of ball $\left(\mathcal{P}_{3}(K)\right)$, while $P_{2}$ is extreme. Indeed, the polynomial $P_{1}$ being real-valued, the second column of the $(2 \times 2)$-matrix $W_{P_{1}}$ is null, whence rank $W_{P_{1}}=1$, whereas the matrix

$$
W_{P_{2}}=\left(\begin{array}{cc}
\mathfrak{R}\left(P_{2}(-1)\right) & \mathfrak{I}\left(P_{2}(-1)\right) \\
\mathfrak{R}\left(P_{2}(1)\right) & \mathfrak{I}\left(P_{2}(1)\right)
\end{array}\right)=\frac{1}{\sqrt{2}}\left(\begin{array}{cc}
1 & 1 \\
-1 & 1
\end{array}\right)
$$

has rank 2 .

Proof of Theorem 2. (ii) $\Longrightarrow$ (i). Suppose (1.1) is fulfilled for some $Q \in \mathcal{P}_{n}$. Then (1.2) holds everywhere on $K$, whence in particular

$$
|Q(x)|^{2} \leq 1-|P(x)|^{2}, \quad x \in K .
$$

Here, the right-hand side is $O\left(\left|x-x_{j}\right|^{m_{j}}\right)$ as $x \rightarrow x_{j}$, and so

$$
Q(x)=O\left(\left|x-x_{j}\right|^{m_{j} / 2}\right) \quad \text { as } x \rightarrow x_{j}, x \in K \text {. }
$$

Since $Q$ is a polynomial, while $\mu_{j}$ is the smallest integer in the interval $\left[m_{j} / 2, \infty\right)$, it actually follows from (2.2) that $Q$ has a zero of multiplicity $\geq \mu_{j}$ at $x_{j}$. Hence

$$
Q(x)=Q_{0}(x) \prod_{j=1}^{N}\left(x-x_{j}\right)^{\mu_{j}}
$$

for some polynomial $Q_{0}$.

Now if $\mu>n$, then (2.3) is only possible for $Q \equiv 0$, which implies that $P$ is an extreme point.

It remains to consider the case where $\mu \leq n$ and $\operatorname{rank} W_{P}=2(n-\mu+1)$. In this case, (2.3) holds for some $Q_{0} \in \mathcal{P}_{n-\mu}$, and we write

$$
Q_{0}(x)=\sum_{k=0}^{n-\mu}\left(c_{k}+i d_{k}\right) x^{k}
$$

with $c_{k}, d_{k} \in \mathbb{R}$. Also, (2.3) yields

$$
\mathfrak{R}(\bar{P}(x) Q(x))=\prod_{j=1}^{N}\left(x-x_{j}\right)^{\mu_{j}} \Re\left(\bar{P}(x) Q_{0}(x)\right) .
$$


Substituting this into the inequality

$$
|\Re(\bar{P}(x) Q(x))| \leq 1-|P(x)|^{2}, \quad x \in K
$$

(which is a consequence of (1.2)), we get

$$
\prod_{j=1}^{N}\left|x-x_{j}\right|^{\mu_{j}}\left|\Re\left(\bar{P}(x) Q_{0}(x)\right)\right| \leq 1-|P(x)|^{2}, \quad x \in K .
$$

The right-hand side of $(2.6)$ being $O\left(\left|x-x_{j}\right|^{m_{j}}\right)$ as $x \rightarrow x_{j}$, we deduce that

$$
\mathfrak{R}\left(\bar{P}(x) Q_{0}(x)\right)=O\left(\left|x-x_{j}\right|^{m_{j}-\mu_{j}}\right) \quad \text { as } x \rightarrow x_{j}, x \in K \text {. }
$$

Here, the restriction $x \in K$ can be actually dropped (i.e., replaced by $x \in \mathbb{R}$ ), since $\mathfrak{R}\left(\bar{P} Q_{0}\right)$ is a polynomial. Thus (2.7) tells us that $\mathfrak{R}\left(\bar{P} Q_{0}\right)$ vanishes at $x_{j}$ with multiplicity at least $m_{j}-\mu_{j}$; of course, this is only meaningful for $1 \leq j \leq M$, since otherwise $m_{j}=\mu_{j}=1$. Therefore,

$$
\mathfrak{R}\left(\bar{P} Q_{0}\right)^{(l)}\left(x_{j}\right)=0 \quad\left(1 \leq j \leq M, 0 \leq l \leq m_{j}-\mu_{j}-1\right) .
$$

With (2.4) plugged in, (2.8) becomes a homogeneous system of linear equations with respect to the unknowns $c_{0}, \ldots, c_{n-\mu}, d_{0}, \ldots, d_{n-\mu}$. The matrix of the system is $W_{P}$, and the hypothesis $\operatorname{rank} W_{P}=2(n-\mu+1)$ ensures that the only solution is the trivial one. Hence $Q_{0} \equiv 0$, which implies $Q \equiv 0$ and proves that $P$ is an extreme point.

(i) $\Longrightarrow$ (ii). Conversely, if $\mu \leq n$ and $\operatorname{rank} W_{P}<2(n-\mu+1)$, then the homogeneous system just mentioned has a nontrivial solution, so that (2.8) holds with some $Q_{0} \in \mathcal{P}_{n-\mu}, Q_{0} \not \equiv 0$. Now if the norm $\left\|Q_{0}\right\|_{\infty}$ is appropriately small (which can be safely assumed), then the nontrivial polynomial $Q \in \mathcal{P}_{n}$ defined by (2.3) will satisfy

$$
|Q|^{2} \leq \frac{1}{2}\left(1-|P|^{2}\right)
$$

and

$$
2|\Re(\bar{P} Q)| \leq \frac{1}{2}\left(1-|P|^{2}\right)
$$

everywhere on $K$. Indeed, for $j=1, \ldots, N$, the left-hand sides of (2.9) and (2.10) vanish at $x_{j}$ with multiplicity at least $m_{j}$ each. (To see why, recall that $2 \mu_{j} \geq m_{j}$ and use the relations (2.5) and (2.8).)

Taken together, (2.9) and (2.10) yield (1.2), and we conclude that $P$ fails to be extreme in ball $\left(\mathcal{P}_{n}(K)\right)$. 


\section{References}

[D] K. M. Dyakonov, Polynomials and entire functions: zeros and geometry of the unit ball, Math. Res. Lett. 7 (2000), 393-404.

[G] A. L. Garkavi, The unit sphere of the space of polynomials with an integral metric, Mat. Zametki 1 (1967), 299-304. (Russian)

[H] K. Hoffman, Banach Spaces of Analytic Functions, Prentice-Hall, Inc., Englewood Cliffs, N. J., 1962.

[KR] A. G. Konheim and T. J. Rivlin, Extreme points of the unit ball in a space of real polynomials, Amer. Math. Monthly 73 (1966), 505-507.

[P] L. D. Parnes, Extreme points of unit balls in polynomial spaces, Functional Analysis, 31, 111-116, Ul'yanovsk. Gos. Ped. Inst., Ul'yanovsk, 1990. (Russian)

[R] H.-J. Rack, Extreme Punkte in der Einheitskugel des Vektorraumes der trigonometrischen Polynome, Elem. Math. 37 (1982), 164-165.

Steklov Institute of Mathematics, St. Petersburg Branch (POMi), Fontanka 27, St. Petersburg, 191011, Russia.

E-mail address: dyakonov@pdmi.ras.ru

Current address:

Departament de Matemàtica Aplicada i Anàlisi, Universitat de Barcelona, Gran Via 585, E-08071 BARCELONA, Spain.

E-mail address: dyakonov@mat.ub.es 